\title{
Dermoscopy of Seborrheic Keratosis-Like Melanoma
}

\section{Benkirane S*, Oukarfi S, Elloudi S, Douhi Z, BayBay H and Zahra Mernissi F \\ Department of Dermatology, University Hospital Hassan II, Morocco}

*Corresponding author: Selma Benkirane, Department of Dermatology, University Hospital Hassan II, Morocco, Tel: 00212661253442; Email: dr.benkiraneselma@gmail.com

\section{Letter to Editor}

Volume 5 Issue 1

Received Date: January 06, 2020

Published Date: January 23, 2020

DOI: $10.23880 /$ cdoaj-16000199

\section{Dear Editor,}

Let me send you a case report of dermoscopic diagnosis of melanoma resembling seborrheic keratosis

77-year-old patient, diabetic on insulin, who consulted for an increase in the size of a pigmented lesion in the left arm for 1 year asymptomatic. The dermatological examination found multiple seborrheic keratoses on the face, and a well limited blackish-brown pigmented plaque, slightly raised by place at the level of the left arm making about $3 \mathrm{~cm}$ of long axis (Figure 1) whose clinical appearance resembled a pigmented seborrheic keratosis because it had prominent pseudofollicular openings on its surface (Figure 2). Dermoscopic examination revealed an asymmetrical and pigmented lesion with a bluish, dark brown to light brown and black coloration demonstrating a clearly visible bluewhite veil on the raised center of the lesion suggesting a malignant melanoma and a rainbow appearance, of more, a well-defined zone of seborrheic keratosis with multiple pseudo comedones (Figure 3). The examination of the lymph nodes was normal. A diagnosis of superficial spread malignant melanoma (MSS) was made histologically. An extension assesment was without particularities. A wide excision with margin of $0.5 \mathrm{~cm}$ was performed.

Melanoma in situ can clinically mimic seborrheic keratosis which is considered an easily diagnosed tumor. Unfortunately, some melanomas can resemble this common tumor, which can delay treatment and worsen the prognosis. In our case dermoscopy was useful to suspect the diagnosis of melanoma keratosis seborrheic like and to perform a skin biopsy for diagnostic confirmation thus showing the importance of the clinical-dermoscopic-histopathological correlation in front of any doubtful melanocytic lesion thus allowing an early detection. As a rule, to avoid missing melanomas, all nodules that cannot be classified into a benign category with certainty should be promptly excised.

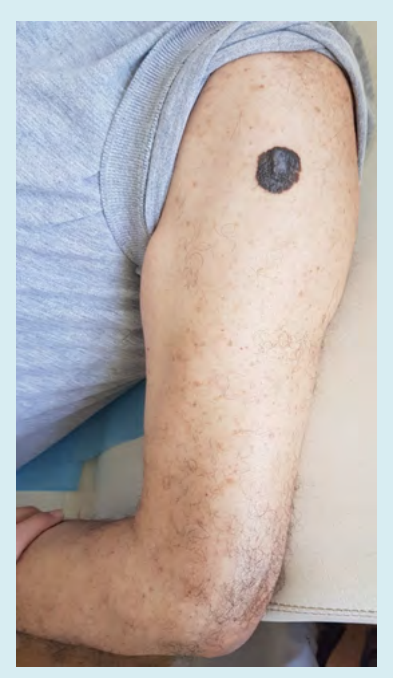

Figure 1: Blackish brown pigmented plaque on the left arm. 


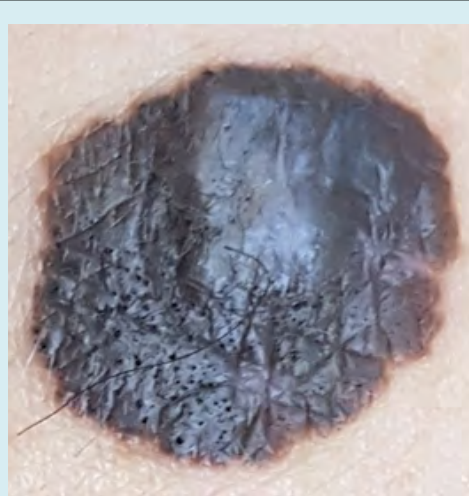

Figure 2: Clinical appearance showing pseudo-follicular openings compatible with the appearance of pigmented seborrheic keratosis.

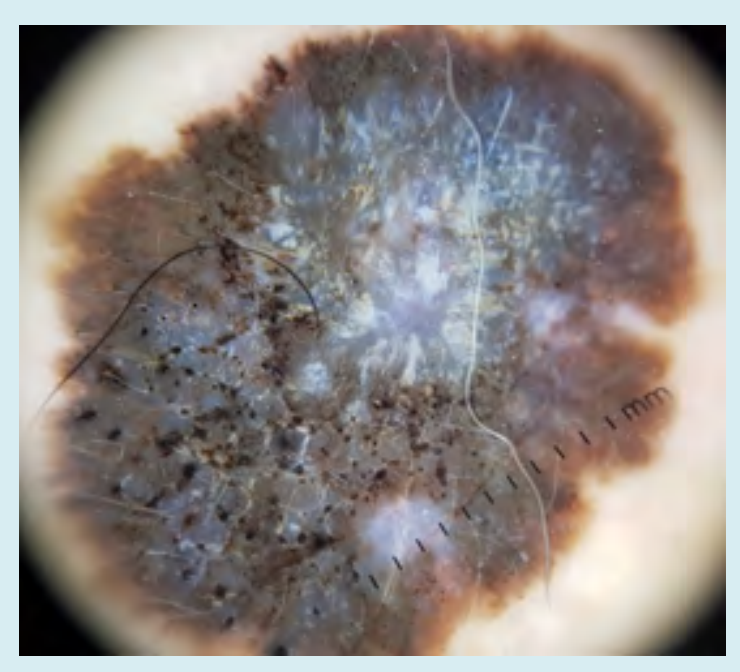

Figure 3: Dermoscopic examination revealed an asymmetrical and pigmented lesion with a bluish, dark brown to light brown and black coloration demonstrating a clearly visible blue-white veil and a rainbow appearance, and multiple pseudo comedones. 